
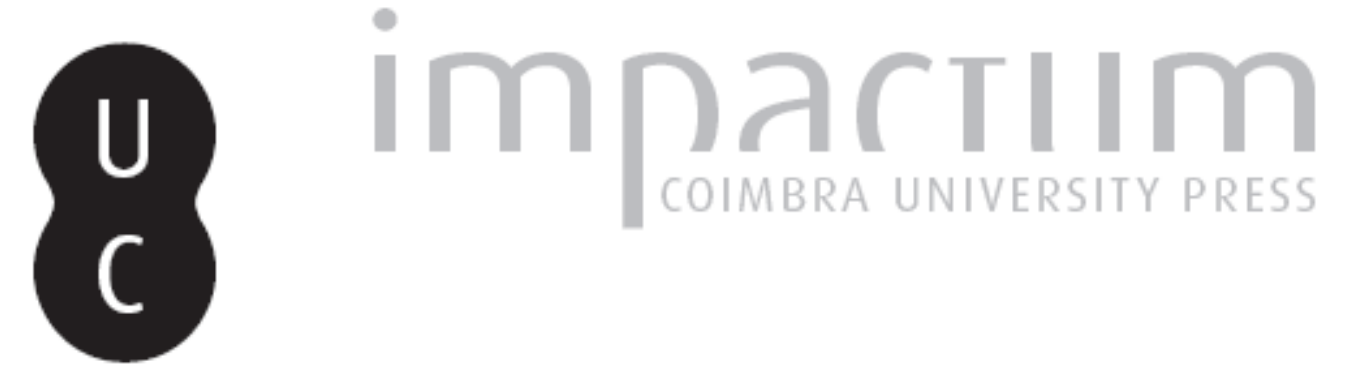

Un regard de médiéviste français sur l'historiographie de la formation du Portugal

Autor(es): Boissellier, Stéphane

Publicado por: Faculdade de Letras da Universidade de Coimbra, Instituto de História Económica e Social

URL persistente:

URI:http://hdl.handle.net/10316.2/27916

DOI:

DOl:http://dx.doi.org/10.14195/0870-4147_42_8

Accessed : $\quad$ 26-Apr-2023 13:11:47

A navegação consulta e descarregamento dos títulos inseridos nas Bibliotecas Digitais UC Digitalis, UC Pombalina e UC Impactum, pressupõem a aceitação plena e sem reservas dos Termos e Condições de Uso destas Bibliotecas Digitais, disponíveis em https://digitalis.uc.pt/pt-pt/termos.

Conforme exposto nos referidos Termos e Condições de Uso, o descarregamento de títulos de acesso restrito requer uma licença válida de autorização devendo o utilizador aceder ao(s) documento(s) a partir de um endereço de IP da instituição detentora da supramencionada licença.

Ao utilizador é apenas permitido o descarregamento para uso pessoal, pelo que o emprego do(s) título(s) descarregado(s) para outro fim, designadamente comercial, carece de autorização do respetivo autor ou editor da obra.

Na medida em que todas as obras da UC Digitalis se encontram protegidas pelo Código do Direito de Autor e Direitos Conexos e demais legislação aplicável, toda a cópia, parcial ou total, deste documento, nos casos em que é legalmente admitida, deverá conter ou fazer-se acompanhar por este aviso.

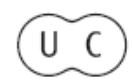





\title{
Un regard de médiéviste français sur l'historiographie de la formation du Portugal
}

\author{
StéPHANe BoissellieR \\ Université de Poitiers \\ Centre d'Etudes Supérieures de Civilisation Médiévale \\ boissell@orange.fr
}

Resumo:

Pour 1'Europe occidentale médiévale, c'est au Portugal que l'apparition politique d'une nation peut être le mieux observée. Cet article tente d'évaluer les apports de l'historiographie portugaise à ce sujet et de suggérer des pistes de recherches pour approfondir les voies déjà tracées, notamment par J. Mattoso. En filigrane, on se demande ce qu'un auteur français, formé par une tradition historiographique spécifique, peut apporter à la compréhension du problème.

Palavras chave:

Historiographie; Ethnogenèse; Portugal.
Abstract:

In the context of medieval Western Europe, Portugal is where ethnogenesis (the political emergence of a nation) can be best observed. This article tries to assess the contributions of Portuguese historiography on this theme and to suggest some research hints in order to deepen the ways already defined, mainly by J. Mattoso. Between the lines, one might ask how a French author, with a specific historiographical education, can contribute to the understanding of this problem.

Keywords:

Historiography; Ethnogenesis; Portugal. 
Les liens des historiens français avec la Faculté des Lettres de l'Université de Coimbra, avec un de ses plus prestigieux Instituts (celui d'Histoire Economique et Sociale, autrefois Institut d'Etudes Historiques), et avec la Revista Portuguesa de História sont anciens et nombreux - même si, chez les médiévistes, ils ne sont plus aussi intenses qu' autrefois, quand mes prédécesseurs prenaient le soin de franciser Coimbra en «Coïmbre». Ce m'est donc un devoir, un plaisir et un honneur de renouer le fil d'une si heureuse collaboration; pour ce faire, et pour rendre hommage au grand maître que fut le Prof. António de Vasconcelos, je me placerai sous le patronage du chanoine Pierre David, dont l'œuvre à Coimbra illustre non seulement la fécondité des échanges scientifiques luso-français ${ }^{1}$ mais aussi l'accueil généreux que le Portugal sut offrir dans les temps douloureux que vivait alors l'Europe.

L'histoire du Portugal est étonnamment ignorée, en France et dans toute l'Europe, dans le public cultivé et même parmi les historiens professionnels ${ }^{2}$, sauf pour ses phases très contemporaines - principalement la dictature salazariste et plus encore la «révolution des œillets» de 1974, sur lesquelles abondent les films, reportages et ouvrages - ou pour quelques épisodes popularisés par de grands littérateurs français (le tremblement de terre de 1755, peint par Voltaire, ou les amours du roi Pierre Ier, ressuscitées par la «Reine morte» d'Henri de Montherlant). Ignorance étonnante, disons-nous, parce qu'aucun peuple n'est plus proche de la France dans l'histoire récente: les Portugais constituent numériquement la plus importante communauté d'origine étrangère en France et également (cependant?) l'intégration la mieux réussie, comme en attestent les nombreuses associations d'amitié et d'échanges franco-portugais, les revues

1 Rappelons que la cathédrale de Coimbra fut un objet commun aux deux grands savants cités dans ce bref préambule.

2 Les quelques ouvrages récents sérieux existant en français sur l'histoire du Portugal sont des histoires générales (des origines à nos jours) comme celles de R. Durand chez Hâtier, J.-F. Labourdette chez Fayard, un «Que sais-je?» (d'A.-A. Bourdon, réécrit par J.-F. Labourdette) aux PUF. Dans le domaine de l'édition universitaire proprement médiéviste, il existe deux manuels (de D. Menjot chez Hachette et A. Rucquoi au Seuil) consacrés à l'histoire médiévale de la Péninsule ibérique, qui incluent, assez marginalement, le Portugal. Sur le Moyen Âge portugais spécifiquement, il n'existe donc pas de synthèse dans aucune autre langue européenne que le portugais - le récent Stephen Lay, The Reconquest kings of Portugal. Political and cultural reorientation of the medieval frontier, New York, Palgrave Macmillan, 2009 est une compilation d'une série de biographies royales, qui ne constitue ni une synthèse ni un véritable essai (voir mon CR dans les Mélanges de la Casa de Velázquez, 40-2 (2010), p. 241-244). 
bilingues et bi-culturelles ${ }^{3}$. Même en remontant aux temps les plus anciens, des liens étroits existent déjà entre les deux pays, puisque l'Etat portugais a été fondé précisément, au début du XIIe siècle, par une dynastie d'origine bourguignonne, et certaines réformes les plus fondamentales du Moyen Âge portugais ont probablement été inspirées au roi Alphonse III par la «formation» qu'il a reçue en France (comme comte de Boulogne et vassal du roi saint Louis). Alors que fleurissent de bons ouvrages en français sur les périodes anciennes de pays beaucoup plus «exotiques», comme la Hongrie ou la Scandinavie vikingue, l'apparition du Portugal en tant que nation n'est éclairée par aucun livre en français, et les Portugais de France (de même que leurs enfants français, dits «luso-descendants») méconnaissent le plus souvent les origines de leur pays et/ou surtout les racines les plus profondes de leur culture.

Pour les Français «de souche», le sens de cette ignorance est différent mais pas moins grave, dans une Europe qui se veut de plus en plus intégrée et pluri-culturelle (en particulier sur le plan linguistique). Il existe pourtant quelques médiévistes français qui se sont intéressés au Moyen Âge portugais, depuis la Seconde Guerre Mondiale, et l'historiographie française a joui d'une certaine faveur dans les milieux académiques portugais, au point que le français reste une langue d'expression parmi nos collègues lusitaniens ${ }^{4}$.

Pour tout historien européen, la naissance politique du Portugal et sa formation culturelle sont ou devraient constituer un problème fascinant. En effet, l'apparition tardive de cette nation médiévale, au XII ${ }^{\mathrm{e}}$ siècle, bénéficie d'un éclairage documentaire beaucoup plus vif que la formation des vieux royaumes «barbares» du début du Moyen Âge. Or, comme le montrent les temps récents, les enjeux des sociétés occidentales se situent depuis très longtemps dans le cadre des Etats-nations; les nations actuelles de l'Europe, dont l'espace a d'abord été de simples provinces relativement uniformes de l'Empire romain, sont indubitablement apparues au Moyen Âge - même si certains processus identitaires peuvent remonter plus loin -, parce que c'est à cette époque, entre le $\mathrm{V}^{e}$ et le XII ${ }^{\mathrm{e}}$ siècle, que se sont formés la plupart des Etats d'Europe occidentale, dont les nôtres sont les descendants par-delà les changements de régimes et de

3 L'inverse est moins net mais tout de même sensible: d'après les chiffres 2004 du Ministère des Affaires Etrangères français, le Portugal figure en $7^{\mathrm{e}}$ place de l'Union européenne comme lieu de résidence des Français expatriés, avec 27000 résidents (mais en fait au $3^{\mathrm{e}}$ rang si l'on prend en compte le chiffre de population des pays d'accueil).

4 Voir la $2^{\mathrm{e}}$ partie de l'article de Bernardo Vasconcelos e Sousa et Stéphane Boissellier, «Pour un bilan de 1'historiographie sur le Moyen Âge portugais au XXe siècle», Cahiers de Civilisation Médiévale, 49 («La médiévistique au XXe siècle, bilan et perspectives») (2006), p. 213-256. 
conceptions politiques ${ }^{5}$. Pour la majorité des pays d'Europe, éclairer le Moyen Âge, c'est donner non pas toutes mais certaines clés de l'identité des Etats-nations contemporains.

L'étude de la formation du Portugal illustre donc un des problèmes essentiels de l'histoire de l'Occident européen. On peut y observer ce que le «complexe de Clovis» interdit d'étudier en France ${ }^{6}$. Au-delà, ce sujet présente un intérêt plus généralement intellectuel et civique, pour tous les Européens. L'adhésion du Portugal à la Communauté européenne a ravivé des interrogations identitaires très actuelles, dont beaucoup sont certes étroitement luso-portugaises (le complexe d'être le parent pauvre de l'Europe, depuis le début du XIX ${ }^{\mathrm{e}}$ siècle, le fait d'être le seul pays réellement méditerranéen dans une position atlantique) mais dont les fondements impliquent la définition même de l'Europe, en ces temps de débat sur l'adhésion de la Turquie à la Communauté européenne: atlantique (autrement dit occidentale, surtout avec le «prolongement» américain de l'Europe) ou méditerranéenne (c'est-à-dire en partie asiatique et africaine) ${ }^{7}$, méridionale ou septentrionale, marginale (comme cap de l'Asie, à la remorque des civilisations du Proche-Orient) ou centrale?

\section{Le royaume portugais: naissance, formation, genèse, identification?}

Un célèbre ouvrage, évoquant l'identité ibérique médiévale écartelée entre Orient musulman et Occident chrétien, s'intitule L'Espagne, une énigme historique ; mais c'est au Portugal que cette formule s'appliquerait le mieux: pays soumis aux influences islamiques et latines et issu de l'affrontement entre ces deux ensembles politico-culturels, comme toute la Péninsule ibérique, il a conquis et maintenu envers et contre tout (surtout contre le León-Castille) son identité culturelle et son indépendance politique au sein du concert ibérique, alors que sa naissance tardive (en tant que royaume) au début du XII ${ }^{e}$ siècle est presque illogique, dans un contexte de centralisation ou au moins de fédération entre les différents royaumes ibériques. En fait, c'est surtout à l'extrême fin du

5 C'est ce qui explique le poids de la recherche sur le Moyen Âge dans les milieux académiques au XIXe siècle.

6 Dans l'historiographie récente, peu d'ouvrages osent encore aborder Les origines de la France (titre d'un ouvrage dont l'auteur, Edward James, est d'ailleurs britannique), sauf en se restreignant à son aspect identitaire, comme le célèbre Naissance de la nation France, de Colette Beaune.

7 La France, qui se veut, le moteur d'une «Union pour la Méditerranée», devrait être particulièrement intéressée par le problème de l'identité portugaise.

8 Claudio Sánchez Albornoz, España, un enigma historico, Buenos Aires, 1956 (2 vol.). 
Moyen Âge, quand toutes les autres nations ibériques fusionnent en un seul bloc, que commence à ressortir plus nettement la force de la personnalité portugaise: de nos jours, c'est encore la seule région au sud des Pyrénées à ne pas faire partie de l'Espagne, véritable affront à une «logique» géographique apparente qui isole (donc unifie) nettement l'Ibérie, en tant que péninsule, sur une carte.

Le Portugal est l'Etat qui s'est formé le plus tardivement au sein dans cet ensemble, mais c'est aussi le territoire qui a la plus ancienne frontière d'Europe; le pays est donc né «tout armé», avec une personnalité déjà constituée ou au moins forgée très rapidement. Bien sûr, la volonté et l'action des rois de la première dynastie ont cristallisé ces éléments durables d'identité, et il ne faut pas méconnaître la dimension politique qui a ordonné les lames de fond de la culture et des structures socio-économiques ${ }^{9}$. Dans la trame événementielle de cette histoire, il y a une part d'aléatoire; prendre les racines profondes de l'identité pour fil conducteur ne doit pas amener à reconstituer chaque moment historique en fonction de ce qui est arrivé plus tard, sous peine de conclure que l'identité portugaise existe depuis toujours et que l'existence du Portugal en tant qu'entité est inévitable.

Ce «miracle» de l'indépendance portugaise n'est pourtant pas celui qui a le plus retenu l'attention des historiens français, surtout médiévistes; pendant longtemps, leur étonnement et leur fascination se sont portés vers le $\mathrm{XV}$ siècle (Robert Ricard, l'africaniste Raymond Mauny), quand ce petit royaume, vieux de trois siècles seulement, a entrepris une expansion territoriale au Maroc, puis, surtout, quand il a élargi cette conquête, sur les mers, à la découverte du monde, par l'exploration des côtes atlantiques de l'Afrique, la découverte du Brésil et l'ouverture de la route des Indes: le grand poète Camões, chantre de cette épopée, qualifie le Portugal de «pays où la terre finit et où la mer commence», et c'est sa conception que les historiens ont retenue, parlant parfois, avec beaucoup d'abus, de la «vocation maritime» du Portuga ${ }^{10}$. C'est d'ailleurs cette phase finale du Moyen Âge portugais, dominée par la figure du prince Henri «le Navigateur», qui est la moins mal connue à l'étranger.

Pourquoi alors s'intéresser à la naissance du Portugal? En la prenant pour objet de réflexion, il ne s'agit pas de réhabiliter une approche dénoncée depuis longtemps comme «mythe des origines», c'est-à-dire la conception totalement

9 Une récente collection de biographies royales («Reis de Portugal», publiée par le Círculo de Leitores) a enfin doté l'historiographie portugaise d'une meilleure appréhension du rôle personnel des dirigeants suprêmes.

${ }_{10}$ Yves Bottineau, Le Portugal et sa vocation maritime. Histoire et civilisation d'une nation, Paris, De Boccard, 1977. 
déterministe selon laquelle chaque phénomène se déroulant dans le temps serait inclus tout entier dans sa genèse, à l'image des sciences dures qui ont longtemps privilégié la nécessité par rapport au hasard pour expliquer les phénomènes matériels: les phénomènes sociaux et culturels observés par l'historien sont trop complexes pour se laisser réduire à une pure mécanique linéaire. L'observation dans le temps long nous montre que ce qui est durable n'est pas pour autant définitif, et que toute l'histoire, fût-elle strictement nationale, n'est pas contenue, même potentiellement, dans quelques «moments-clés»: Fernand Braudel, étudiant L'identité de la France, dut balayer un spectre chronologique allant de la Préhistoire à la première Guerre Mondiale pour rassembler tous les caractères qui ont été distinctifs de l'identité à un moment ou à l'autre de la construction de la France. Et l'on pourrait à la limite bannir le concept même de «genèse», en avançant que celle-ci est simplement une combinaison un peu plus inhabituelle parmi toutes les façons dont se combinent constamment les facteurs «de longue durée» d'évolution des sociétés.

La chronologie des premiers siècles de l'histoire portugaise nous montre bien la nécessité de distinguer et réunir à la fois ce qui relève de la genèse et ce qui relève de l'évolution sur la longue durée. Durant un premier siècle, de 1130 à 1250, le royaume portugais est dominé par la Reconquête contre les Maures (phénomène exclusivement ibérique) et par son désir d'indépendance (phénomène intrinsèque); cette phase de formation est celle de la plus grande originalité, mais toutes les structures de cette époque ne sont pas durables (ainsi, l'économie guerrière, la fluidité sociale, la domestication de l'Eglise par la monarchie, l'absence d'idéologie féodale dans l'aristocratie). En revanche, entre 1250 et 1380, le Portugal perd de nombreuses spécificités, notamment parce qu'il s'ouvre aux courants culturels traversant toute l'Europe, mais les structures alors mises en place dureront jusqu'aux bouleversements du XIX ${ }^{\mathrm{e}}$ siècle (une forte administration étatique, comparable à celle des Capétiens, une Eglise plus nettement liée à Rome, une économie à dominante rurale, une noblesse de plus en plus proche du trône...)

Les quelques décennies ou siècles de formation d'un Etat ne contiennent donc pas toute l'évolution future jusqu'à nos jours. Mais tout de même, l'apparition d'une structure politique propre a constitué un fait décisif pour les hommes qui vivaient à l'extrémité centro-occidentale de la Péninsule ibérique. Même si l'évolution ne s'est pas arrêtée au début du XVI $\mathrm{XI}^{\mathrm{e}}$ siècle et nous éloigne toujours plus des racines anciennes, des évolutions essentielles ont eu lieu durant le millénaire médiéval. C'est d'autant plus vrai pour le Portugal, dont le territoire actuel (autrement dit l'espace matériel ou, mieux, la «zone d'action») s'est cristallisé entre 1130 et 1297 et n'a quasiment plus évolué depuis: bel exemple 
de «racines médiévales»... Les livres majeurs sur le Moyen Âge portugais, depuis A. Herculano au XIX ${ }^{\mathrm{e}}$ siècle $^{11}$ jusqu'à J. Mattoso ${ }^{12}$, ont d'ailleurs pris pour objet les premiers temps de l'histoire nationale et non pas les pages brillantes de l'expansion outre-mer, qui ont certes pesé lourd dans le destin et dans la renommée du Portugal mais n'ont peut-être pas orienté aussi décisivement son identité culturelle que l'action de la première dynastie.

Bien sûr, comme nous le rappelle récemment Patrick Geary, les «nations» médiévales n'ont pas grand-chose à voir avec les Etats-nations qui se sont construits à partir du XIX ${ }^{\mathrm{e}}$ siècle, et qui ont précisément instrumentalisé leur passé le plus lointain pour légitimer les formes qu'ils étaient en train de prendre (territorialité rigoureuse, uniformisation linguistique, adhésion explicite à un pouvoir par un «pacte social», parfois même définition raciale... $)^{13}$; en écrivant une «histoire de... [tel Etat contemporain]», le risque d'anachronisme est d'autant plus grand que les méthodes historiques elles-mêmes, que nous utilisons encore, sont nées précisément avec les Etats-nations ${ }^{14}$. Décrire la progressive constitution d'un ensemble d'individus et de groupes, en une communauté homogène, sur un espace donné, durant les temps médiévaux, n'équivaut donc pas forcément à écrire l'histoire d'une nation actuelle - démarche téléologique presque inévitable, quand, au lieu de se concentrer sur telle époque particulière pour elle-même, on écrit une «histoire de... [tel Etat contemporain] des origines à nos jours» (entité qui se définit, dans une démarche régressive, par le territoire et les structures actuels). Certes, avec les plus anciennes frontières d'Europe dont s'enorgueillit le Portugal, il est un peu moins absurde que pour la France ou l'Italie d'écrire une «histoire du Portugal» (i.e. de tous les faits étant advenus autrefois dans le territoire actuel) incluant le Moyen Âge; mais l'attention toute spéciale que mérite la formation territoriale du Portugal ne doit pas être dans le même esprit qu'à l'époque où fleurissaient en Europe les chaires universitaires

11 Alexandre Herculano, História de Portugal desde o começo da monarquia até o fim do reinado de Afonso III. Notas críticas de José Mattoso, Lisboa, Livraria Bertrand, 1980-1981 (2e-4e éd. 1862-1876) (4 vol.); la $1^{\mathrm{e}}$ éd. date de 1846-1853.

12 José Mattoso, Identificação de um País. Ensaio sobre as origens de Portugal 1096-1325, Lisboa, Editorial Estampa, 1988 (3 éd.) (2 vol.).

13 Patrick J. Geary, Quand les nations refont l'histoire. L'invention des origines médiévales de l'Europe, Paris, Aubier, 2004.

14 Ainsi en va-t-il de notre critique des chroniques anciennes, que nous savons être des légitimations de leurs commanditaires, en fait presque toujours des écrits purement «politiques» (légitimant l'exercice d'un pouvoir), alors qu'elles sont souvent principalement des mises en scène symboliques, voire mythologiques, de leurs acteurs (en tant qu'individus, que chrétiens ou que membres d'une parenté et non pas forcément en tant que détenteurs de l'autorité) 
de «géographie historique» de chaque nation ${ }^{15}$. D'ailleurs, l'adéquation entre le rex (roi, donc construction politique), la gens (peuple, mais il vaudrait mieux dire «race», donc identité ethnique) et la patria (notion plutôt territoriale) n'a pas été inconnue dans l'Espagne wisigothique ${ }^{16}$.

L'exemplarité du Portugal, comme nation médiévale et comme résumé de l'Europe actuelle, a ses limites. Mais, à l'inverse, le «destin exceptionnel» n'est qu'un mythe, quand il s'applique aux nations: l'histoire d'un groupe humain inclut une telle somme de faits que l'originalité y est forcément irréductible, par le simple effet de la statistique. C'est un mérite majeur des recherches récentes d'avoir montré que, au Moyen Âge, tout est différent partout (jusque et surtout à l'échelle locale), mais que les grands traits de civilisation de l'Occident se retrouvent partout aussi - manière de voir qui n'est d'ailleurs pas sans relation avec la construction de l'Union Européenne... Il me semble d'ailleurs que les historiens portugais ont été moins soucieux que leurs confrères «espagnols» (parlant en fait le plus souvent au nom de toute la Péninsule ibérique) de faire du Portugal un pays à part au sein de l'Europe ${ }^{17}$ - même si la devise «orgueilleusement seuls» de la propagande salazariste répond à l'España es diferente. Plus précisément, la «portugalité», si l'on peut dire, est fortement affirmée par les études historiques depuis le XVIIe siècle, mais elle vise surtout à se différencier du pays voisin ${ }^{18}$; c'est seulement pour l'époque où leurs princes ont lancé les «caravelles du Christ» à la découverte du monde que, dans un élan de fierté, les historiens portugais des XIX-XXe siècles ont jugé «sans pareil» le destin de leur pays ${ }^{19}$ - jusqu'à se rendre compte, quand sont perdues les dernières colonies, que définir un pays par son Empire est une duperie identitaire.

15 Voir Pierre Toubert, «Frontière et frontières: un objet historique», in Castrum 4. Frontière et peuplement dans le monde méditerranéen au Moyen-Age. Actes du colloque d'Erice-Trapani (Italie) tenu du 18 au 25 septembre 1988, Rome-Madrid, Ecole française de Rome / Casa de Velázquez, 1992, p. 9-17.

16 Ce fait a bien été mis en lumière par Suzanne Teillet, Des Goths à la nation gothique. Les origines de l'idée de nation en Occident du Ve au VIIe siècle, Paris, Les belles lettres, 1984, ouvrage que ne semble pas utiliser P. Geary, Quand les nations refont l'histoire, cit.

17 En tout cas, le regard des autres accorde - à tort d'ailleurs - moins de personnalité au Portugal qu'au pays voisin: dans l'imaginaire des Européens, on n'a pas, pour le Portugal, les fantasmes de Carmen, de la tauromachie et des «contes de l'Alhambra» qui caractérisent - en la défigurant ! - l'Espagne.

18 Certes, l'affirmation d'une inexistence de la féodalité, censée marquer une exception globalement ibérique, a eu sa place au Portugal comme en Espagne.

19 Le Portugal «eut comme grand dessein national de transplanter son organisme dans d'autres régions du monde... et fit du sentiment œcuménique la raison de son existence... De telles valeurs constituent un motif suffisant pour graver l'action portugaise dans le patrimoine 


\section{Courants historiographiques}

Les origines du Portugal ont été diversement interprétées par les historiens, et en premier lieu, bien sûr, par les historiens portugais eux-mêmes, qui se sentaient et se sentent encore directement concernés par cette histoire ${ }^{20}$. Sans remonter trop loin, on peut dire que l'historiographie de la naissance du Portugal a reposé depuis les années 1930 jusqu'aux années 1970 sur les thèses, dynastiques et géostratégiques, de Damião Peres et Torquato de Sousa Soares (celui-ci introduisant des considérations socio-économiques); puis, depuis 1985, les médiévistes suivent l'essai magistral de José Mattoso, qui a mis en lumière, d'une part l'opposition entre un nord féodal (ou féodalisé) «fondateur» et un sud monarchique conquis et colonisé à partir du précédent (opposition qui recoupe des données géographiques dégagées par Orlando Ribeiro), d'autre part le rôle de l'aristocratie dans la première structuration du pays ${ }^{21}$.

Ce dernier ouvrage, admirable, date de plus de 25 ans, alors que la recherche historique portugaise a précisément connu durant ce délai une véritable

de l'humanité» (Joaquim Veríssimo Serrão, História de Portugal. Volume I Estado, pátria e nação (1080-1415), Lisboa, Verbo, rééd. 1979, p. 16).

$20 \mathrm{Nul}$ ne peut prétendre maîtriser toute l'historiographie médiévistique sur le Portugal; au-delà d'innombrables travaux scientifiques de détail, quelques grandes synthèses offrent une forte capacité de suggestion ou au moins une étape dans la réflexion. Un récit événementiel bien informé se trouve dans Joaquim V. Serrão, História de Portugal, cit.; chronologique et assez scolaire (conçu comme un manuel) mais plus problématique que le précédent, avec certaines mises au point qui restent pénétrantes, est A. H. de Oliveira Marques, História de Portugal desde os tempos mais antigos até à presidência do Sr. general Eanes, Lisboa, Palas editores, rééd. 1985; on peut y joindre les vol. 2 et 3 de José Hermano Saraiva (dir.), História de Portugal, Lisboa, Alfa, 1983, qui sont extrêmement inégaux. Mais ce sont les volumes «médiévaux» de deux grandes collections qui, ayant renouvelé les approches, fournissent à la fois des cadres d'analyse stimulants et de nombreuses données: si le vol. II de la «Nova história de Portugal» (A. H. de Oliveira Marques (dir.), Portugal das invasões germânicas à «Reconquista», Lisboa, Editorial Presença, 1993) est décevant, le vol. III est en revanche excellent, quoique inégal (Maria Helena Coelho et Armando Luís de Carvalho Homem (coord.), Portugal em definição de fronteiras (1096-1325). Do condado portucalense à crise do século XIV, Lisboa, Editorial Presença, 1996); cet ouvrage est toutefois concurrencé par le vol. II de l'«História de Portugal» (José Mattoso (dir.), A monarquia feudal (1096-1480), Lisboa, Círculo de leitores, 1993), qui actualise et systématise les thèses de l'essai capital de José Mattoso, Identificação, cit., tandis que le vol. I de cette même collection constitue la meilleure mise au point sur le haut Moyen Âge portugais (José Mattoso (dir.), Antes de Portugal, Lisboa, Círculo de leitores, 1992).

${ }_{21}$ Damião Peres, Como nasceu Portugal, Porto, 1939 (constamment réédité), Torquato de Sousa Soares, Reflexões sobre a origem e a formação de Portugal, Coimbra, Fac. de Letras, 1962 et Formação do estado português (1096-1179), Livraria editora Sólivros de Portugal, 1989, José Mattoso, Identificação, cit.. 
explosion de ses travaux ${ }^{22}$, que les rééditions et actualisations successives du livre de J. Mattoso n'ont pas pu intégrer totalement. De plus, comme le font remarquer les auteurs de la dernière synthèse problématique d'histoire du Portugal $^{23}$, l'abondance et la spécialisation mêmes des recherches des vingt dernières années, dans un contexte épistémologique redevenu positiviste (raisonnablement), ont quelque peu occulté les débats d'interprétations générales de l'histoire portugaise. Il serait donc utile de redonner un peu de sens à une «histoire en miettes».

Si l'on examine un peu plus en détail l'historiographie du sujet, on peut dire que les interprétations sont de trois types ${ }^{24}$. La première, qui a prévalu depuis le Moyen Âge jusqu'à la fin du XIX ${ }^{\mathrm{e}}$ siècle est purement «politicienne»: le Portugal est né des décisions quasiment arbitraires de princes (les rois léonais qui érigent en provinces les régions au sud du Minho au $\mathrm{X}^{\mathrm{e}}$ siècle, le roi Alphonse VI qui regroupe ces provinces et les confie à un gouverneur, enfin le fondateur du royaume qui obtient l'indépendance et ses successeurs qui la maintiennent) ${ }^{25}$, ce qui fait de ce pays un «hasard historique», selon le mot (reçu comme péjoratif) de Claudio Sánchez Albornoz; et, selon l'historiographie dynastique classique, la nation continue ensuite à être construite par ses rois, désormais autonomes ${ }^{26}$. La seconde, qui se dessine lentement depuis le XVII ${ }^{\mathrm{e}}$ siècle et s'affirme à l'époque romantique, avant de triompher dans la première moitié $\mathrm{du} \mathrm{XX}^{\mathrm{e}}$ siècle et de subsister implicitement dans beaucoup de synthèses, est essentialiste: prenant pour une cause ce qui n'est qu'un produit, c'est un (assez mystérieux et mystique) «esprit national» ou une identité - le sentiment d'appartenance à une communauté et l'attachement à un territoire - qui aurait forgé le «peuple» portugais; plus profondément encore, depuis Oliveira Martins jusqu'à Miguel Torga en passant par António Sérgio et Jaime Cortesão, ces auteurs cherchent à cerner «l'essence» du Portugal ou une «âme» portugaise (le Volksgeist, le génie propre de chaque peuple) - et les analyses de la mythique saudade ne sont pas pour rien dans ce psychologisme. La dernière interprétation, développée depuis

22 Bernardo Vasconcelos e Sousa et Stéphane Boissellier, «Pour un bilan...», cit.

${ }^{23}$ Rui Ramos, Bernardo Vasconcelos e Sousa, Nuno Gonçalo Monteiro, História de Portugal, Lisboa, A Esfera dos livros, 2009, p. II.

24 Naturellement, depuis le début des études historiques méthodiques, au début du XIXe siècle, ces thèses ne s'excluent pas et sont souvent présentes dans un même ouvrage, mais avec plus ou moins de relief.

${ }_{25}$ C'est, si l'on peut dire, l'interprétation «purement historique», c'est-à-dire d'une histoire réduite à l'érudition.

${ }^{26}$ La première grande histoire générale du Portugal, entreprise au XVII ${ }^{\mathrm{e}}$ siècle, témoigne de cette conception personnaliste de la construction des nations, puisqu'elle s'intitule Monarquia Lusitana (et non pas Reino Lusitano). 
le début du XXe siècle par l'ethnographie puis par les géographes, et qui est la plus en vogue actuellement, est culturaliste: la rencontre de multiples facteurs «objectifs» d'identité (échappant à la conscience des acteurs de l'époque et pouvant être totalement indépendants d'eux), tels que la position géographique ou les spécificités des structures sociales, avec une volonté politique et une conscience culturelle a provoqué la particularité d'une population et d'un espace portugais.

Ce dernier courant a le mérite de mieux prendre en compte la complexité et d'être le plus ouvert, mais il tombe parfois dans un relativisme «politiquement correct» et fort anachronique. Certes, le Portugal naît en se coupant d'une Galice dont il partage la langue et les paysages, et ceci pour intégrer (pour les deux tiers de son territoire !) une population étrangère (andalouse); et la diversité qui fonde le nouveau royaume est incontestablement une richesse. Mais elle ne l'est qu'à travers la complémentarité et la convergence des différences: au Portugal comme dans tout l'Occident, la viabilité à long terme de la nation - sans parler du nationalisme - se fonde sur une cohésion sociale, construite non sans dommages collatéraux (non seulement l'abandon de la religion musulmane et de la langue arabe, mais aussi la soumission des communautés de colons au féodalisme, la domestication de l'aristocratie par la monarchie...).

Les thèses culturalistes sont forcément les plus fructueuses, car ce sont elles qui mettent le plus en jeu des problématiques spécifiquement temporelles, donc historiques (le choix des échelles chronologiques, les notions de rythme d'évolution et de rupture). Pour un Etat né au début du XII siècle seulement, il existe une sorte de complexe d'infériorité, qui pousse à rechercher des éléments d'identité jusque dans la plus lointaine antiquité: le principal problème est donc chronologique. Le débat essentiel à cet égard a donc opposé depuis le XIX ${ }^{\mathrm{e}}$ siècle les thèses de la rupture et de la continuité. Pour les uns (depuis Alexandre Herculano jusqu'à T. de S. Soares, en passant par le plus illustre défenseur de cette thèse, l'Espagnol C. Sánchez Albornoz ${ }^{27}$ ), l'invasion musulmane en 711 et la création consécutive d'un «désert stratégique» entre le royaume chrétien des Asturies et al-Andalus constitueraient une rupture politique décisive, opposant de part et d'autre du Douro une Espagne septentrionale de la continuité

27 Si le grand historien espagnol reste le plus brillant représentant de cette thèse - et qui ne risque pas d'être supplanté, car il a été aussi le dernier à la défendre -, tous les médiévistes ibériques s'accordent à rendre hommage à l' «Histoire du Portugal» d'Herculano comme étant la première étude critique sur la question; ce point est à souligner car la médiévistique portugaise, parfois à juste titre mais souvent aussi à cause de sa faible diffusion, est rarement considérée comme pionnière dans les études historiques hispaniques. 
(de tradition wisigothique) à une Espagne méridionale de la rupture (devenue provisoirement andalouse et «orientale»).

Ce problème est d'autant plus sensible pour le Portugal que le territoire du royaume est presque totalement exclu de la zone de plus nette continuité politique - il n'a pas, contrairement au royaume d'Asturies-León, à la Navarre et à l'Aragon, et même à la Castille (née elle aussi du León), de «berceau» montagneux asturien/cantabrique ou pyrénéen ${ }^{28}$. Faisant fi de la capacité de résistance des formations culturelles, ces historiens estiment que la légitimité portugaise ne peut pas remonter au-delà du VIII ${ }^{e}$ siècle. Pour les autres médiévistes (depuis Henrique da Gama Barros et l'ethnographe José Leite de Vasconcelos jusqu'à J. Mattoso), maintenant très majoritaires, il n'y a jamais eu de désertion totale, et la continuité l'emporte donc partout - mais il faut noter que, sous un régime salazariste (1932-1974) fortement si ce n'est agressivement nationaliste, certains historiens ont défendu cette conception «continuiste» pour étayer l'idée d'un Portugal éternel, assimilé à la «race lusitanienne», dans une conception essentialiste des peuples.

On voit donc que le problème de l'identité portugaise, au Moyen Âge, s'inscrit dans le contexte, plus largement ibérique et méditerranéen, de la rencontre et la confrontation entre les types d'organisation sociaux et culturels romano-chrétien et arabo-musulman. Or, les positions des écoles historiques mettent en rapport la problématique politique et la problématique culturelle; à cause de l'importance de la guerre entre Etats durant le Moyen Âge ibérique, ces cultures ont longtemps été présentées comme deux blocs, nettement distincts et donc forcément antagonistes ou incompatibles - les historiens modernes suivant en cela les chroniques rédigées à partir de la fin du $\mathrm{IX}^{\mathrm{e}}$ siècle et surtout au $\mathrm{XII}^{\mathrm{e}}$ siècle -; ensuite, on a tenté de minimiser les oppositions, soit en insistant sur la «tolérance» (convivencia) des protagonistes, soit en minimisant les différences elles-mêmes ${ }^{29}$. Curieusement, le poids respectif des deux cultures dans l'identité nationale a suscité beaucoup moins de polémiques au Portugal que dans la voisine Espagne, où se sont opposés C. Sánchez Albornoz et Américo Castro; une fois encore, on peut voir là un effet, pour une fois heureux,

28 Mais on verra que cette continuité politique est de toute façon une pure fiction.

29 Au-delà des spécificités qu'imposent la langue et la religion, les négationnistes de l'identité andalouse, appuyés par certaines études archéologiques (comme celles, au Portugal, développées par le Campo arqueológico de Mértola), insistent sur l'unité «méditerranéenne» des cultures en présence dans la Péninsule ibérique - ce qui est un peu abusif pour une culture arabe née entre Mer Rouge et Océan Indien - et sur une commune imprégnation gréco-romaine des Arabes, Berbères et Hispaniques, voire sur la «tradition» d'orientalisation du Portugal par les Phéniciens et Byzantins. 
de l'idéologie salazariste, qui, assumant les restes d'un empire colonial, a marié son catholicisme intransigeant avec la thèse irénique (et peut-être pas totalement infondée) d'une tendance naturelle des Portugais au syncrétisme culturel et au mélange racial et d'une vocation à l'universalité ${ }^{30}$. Si le temps de ces grandes polémiques philosophiques sur «l'âme portugaise» n'était pas révolu, c'est actuellement que l'on pourrait attendre un débat sur ce thème, après les thèses de Pierre Guichard soulignant les fortes différences (quoique sans incompatibilité totale) entre structures sociales «orientales» d'al-Andalus et «occidentales» des royaumes chrétiens ibériques ${ }^{31} \ldots$

Par ailleurs, la région «fondatrice» du Portugal (entre les fleuves Minho/ /Lima et Douro), ibérique par sa localisation, est née comme entité propre d'une scission d'avec le royaume de Castille-León; cette origine insurrectionnelle et «adultérine» et la résistance opiniâtre du Portugal à l'unification de la Péninsule - qui a fini par triompher partout ailleurs, en rassemblant la Castille, la Couronne d'Aragon et la Navarre -, mêlées à des considérations géographiques, ont poussé certains à se demander si l'identité profonde du Portugal est réellement aussi «ibérique» que dans les autres royaumes médiévaux et si le destin du Portugal ne se trouve pas dans la conquête de l'Atlantique, autrement dit en tournant le dos au reste de la Péninsule ${ }^{32}$.

${ }^{30}$ Le problème a plus été posé en termes philosophiques généraux que sur la base de données historiques, car l'étude sérieuse du «Portugal» musulman a démarré très récemment; la plupart des arguments érudits étaient fournis par des études espagnoles, faute d'une école portugaise d'arabisants et d'islamisants. Sur la courte histoire de l'arabisme portugais, voir, en français, Eva Maria Von Kemnitz, «Les études arabes du Portugal», Rocznik Orientalistyczny, 46 (1988), p. 79-95; plus actualisés sont Adel Sidarus, «Linguas e civilizações extra-europeias na Universidade portuguesa», in Helena Saianda (éd.), Actas do II encontro nacional da APOCLES, Universidade de Évora, 1998, p. 163-167 et «Um projecto português sobre o Gharb al-Andalus», in Adel Sidarus (éd.), Fontes da história de al-Andalus e do Gharb, Lisboa, IICT - Centro de Estudos Africanos e Asiáticos, 2000, p. 21-32.

31 Pierre Guichard, Structures sociales «orientales» et «occidentales» dans l'Espagne musulmane, Paris-La Haye, Mouton, 1977. Cet auteur étudie surtout les structures familiales (qui sont un élément fondamental des sociétés anciennes), mais d'autres, dans une approche marxiste, insistent sur les différences socio-économiques, opposant de façon un peu simpliste une structure «féodale» chrétienne et une structure «tributaire» andalouse (voir surtout Reyna Pastor, Del Islam al cristianismo. En las fronteras de dos formaciones económico-sociales: Toledo, siglos XI-XIII, Barcelona, Ediciones Península, 1975).

32 C'est la position de Joaquim Veríssimo Serrão, História, op. cit., p. 14, pour qui le Portugal ne devient un «pays», après avoir été une «nation», qu'à partir de l'expansion outre-mer, «qui révéla alors sa profonde dimension ultra-marine». 


\section{De nouvelles voies d'approche?}

Depuis longtemps, l'historiographie européenne s'est globalisée: ce qu'il y avait de spécifique et de fructueux dans l'historiographie française a été approprié, adapté, perfectionné... par les historiens des autres pays; un médiéviste français travaillant sur des cultures étrangères ne peut donc plus apporter qu'une certaine distanciation - ce qui est déjà important...

Si le dossier de la formation du Portugal mérite d'être encore travaillé, c'est précisément dans le but de dégager plus nettement les mécanismes, socio-politiques et culturels, qui ont conféré rapidement et durablement au royaume une spécificité, une unité et une autonomie au sein d'une Péninsule ibérique souvent considérée comme une unité. Pour expliquer l'existence du Portugal en tant qu'entité, il faut étudier les phénomènes de combinaison et d'inter-relations, autrement dit d'intégration, qui animent la population: entre $\mathrm{X}^{\mathrm{e}}$ et $\mathrm{XIII}{ }^{\mathrm{e}}$ siècle, la «digestion» sociale succède à l'absorption territoriale de la Reconquête. Ces forces s'exercent et nous sont perceptibles à l'échelle locale, mais leur ensemble constitue le principal moteur de la construction du Portugal; c'est leur articulation qui comprend la plus grande part d'incertitude, qui force l'historien à l'hypothèse généralisatrice et qui fait de toute étude sur ce sujet un essai.

En assimilant les connaissances acquises par la recherche en cours, il faut reformuler des problèmes de façon plus suggestive que ne l'ont fait la critique factuelle et les interrogations de «sens commun» de l'historiographie érudite. Il serait de peu d'intérêt de produire un récit détaillé des événements politiques, dynastiques et militaires - en d'autres termes de réécrire Herculano, même si son travail ne se limite pas à cela -: c'est là le travail du chroniqueur, qui construit une mémoire, et non pas de l'historien, qui produit une analyse. La vocation de ce dernier, y compris en histoire «nationale» (qui a été le théâtre de tant de constructions purement mémorielles), est de réfléchir sur le rapport entre les traces produites par le passé et ce passé lui-même.

Pour réaliser cette ambitieuse histoire-problème, il faut synthétiser la récente mais très riche historiographie développée par les médiévistes portugais. Fondée sur des recherches longtemps paralysées, en quantité peut-être plus qu'en qualité, par la pensée unique de l'Estado Novo (la dictature salazariste), la réflexion sur la naissance politique et culturelle du Portugal doit fuir le néo-positivisme et progresser par hypothèses. Cette démarche n'est pas prématurée, comme disent ceux qui se réfugient dans l'érudition, car c'est ainsi qu'avance la recherche - en donnant des cadres interprétatifs à l'accumulation 
des matériaux ${ }^{33}-$; il n'en demeure pas moins que l'information reste déséquilibrée, car, jusqu'en 1974, les historiens portugais ont eu tendance à revenir constamment sur les mêmes faits, tenus pour les «heures de gloire» du Portugal, et à laisser dans l'ombre des pans entiers de leur passé médiéval. Les interprétations proposées sont donc inégalement fondées, mais, même sans déséquilibre documentaire, c'est la loi du genre: peut-il en être autrement quand on brasse des mouvements et des événements qui ont affecté l'existence de plusieurs millions d'individus sur plusieurs siècles?

Pour des raisons méthodologiques, il me semble que l'accent doit être mis, de façon plus prononcée, sur des problèmes territoriaux - malgré le rôle du géographe Orlando Ribeiro dans la réflexion sur l'identité portugaise, la géo-histoire reste un des rares domaines où l'historiographie française peut encore revendiquer une certaine avance méthodologique et conceptuelle ${ }^{34}$.

${ }^{33}$ Ce postulat constitue une remise en cause de l'histoire quantitative ou plutôt d'un mauvais usage de la quantification et de l'exhaustivité dans la collecte des sources (et donc du privilège conféré aux données présentes le plus massivement dans les sources); en effet, la «religion du chiffre» a conduit les historiens de l'économie et de la société, surtout dans les années 1950-1980, à oublier un principe bien simple, à savoir que la documentation conservée est un miroir déformant (et pas seulement terni) de ce qui a existé, surtout avant le très bas Moyen Âge (Cf. Arnold Esch, «Chance et hasard de transmission. Le problème de la représentativité et de la déformation de la transmission historique», in. Jean-Claude Schmitt et Otto Gerhard Oexle (dir.), Les tendances actuelles de l'histoire du Moyen Âge en France et en Allemagne. Actes des colloques de Sèvres (1997) et Göttingen (1998) organisés par le CNRS et le Max-Planck-Institut für Geschichte, Paris, Publications de la Sorbonne, 2002, p. 15-29). Si l'on envisage les sociétés anciennes comme des systèmes, dont il convient de comprendre la physiologie plus que l'anatomie, le dépouillement et le comptage de tous les actes qui subsistent (souvent par milliers) n'apportent souvent pas plus d'intelligibilité qu'une unique allusion échappant à un scribe, qu'une donnée archéologique encore isolée ou qu'un écrit inhabituel, épave d'une série disparue: le nombre des témoignages ne fait donc pas leur force, et l'historien doit de ce fait élaborer des modèles, forcément hypothétiques au regard de la documentation conservée, modèles dans lesquels le poids des données n'est pas proportionnel à leur volume. Ce problème est particulièrement sensible pour une région comme le Portugal médiéval, dont la documentation écrite conservée est peu diversifiée avant le $\mathrm{XV}^{\mathrm{e}}$ siècle, interdisant de jouer sur la complémentarité des témoignages.

${ }^{34}$ Préfigurée par la géographie historique dès la fin du XIX ${ }^{\mathrm{e}}$ siècle, mais inaugurée par Fernand Braudel et théorisée (pour les médiévistes) par Charles Higounet («La géohistoire», in Charles Samaran (dir.), L'histoire et ses méthodes. Recherche, conservation et critique des témoignages («Encyclopédie de La Pléiade» XI), Paris, Gallimard, 1961, p. 68-91), et enfin reprise actuellement sur de nouvelles bases conceptuelles - je me permets de renvoyer à Stéphane Boissellier, «Introduction à un programme de recherches sur la territorialité: essai de réflexion globale et éléments d'analyse», in. Stéphane Boissellier (éd.), De l'espace aux territoires: la territorialité des processus sociaux et culturels au Moyen Âge. Actes de la table-ronde des 8-9 juin 2006, CESCM (Poitiers), Turnhout, Brepols, 2010, p. 5-85. 
Cette démarche est imposée par les sources de l'histoire médiévale portugaise, dont la typologie (sinon la quantité, tout à fait appréciable) est restreinte: les chroniques sont rares et stéréotypées jusqu'au XIVe siècle, les sources proprement administratives (recensements, comptabilités) ne sont quasiment pas conservées pour l'ensemble du Moyen Âge, et les sources de la pratique, fort nombreuses, elles (quoique très inégalement selon les régions), offrent une typologie focalisée sur la probation des droits de propriété et de juridiction (avec une énorme domination des actes de propriété et des chartes de franchises et d'immunité), car les registres les plus riches (notariaux ou judiciaires ou de l'administration civile locale) ne sont pas conservés. Beaucoup de phénomènes politiques, socio-économiques et culturels affectant l'ensemble de la société ne nous sont donc accessibles qu'à travers leurs conséquences sur l'organisation spatiale ${ }^{35}$.

Ayant rédigé récemment un essai de réflexion dans la longue durée sur les ressorts de l'espace portugais ${ }^{36}$, je n'insisterai pas sur ce point. Notons seulement que la réflexion des médiévistes a porté largement sur les frontières du royaume - parce qu'étant les plus anciennes d'Europe-, donc dans l'optique du volontarisme politique des princes; or, les frontières ne sont que des cadres, placés à certains endroits à l'issue de processus de polarisation. Quand l'infant Alphonse Henri prend le titre de roi, en 1139, sa juridiction s'étend sur un espace qui n'est pas totalement arbitraire, dans la mesure où il est défini (grossièrement) par son «contenu» matériel et humain. Ces processus de territorialisation, combinant l'échelle locale et des niveaux «macro», sont évidemment trop lents pour ne pas avoir précédé l'indépendance politique ${ }^{37}$.

L'histoire est une science humaine et sociale: plus que la terre portugaise (qui relève, en tant que telle, de l'étude géographique), c'est le peuple

${ }^{35}$ C'est d'ailleurs une démarche qui ne devrait pas être réservée aux zones moins privilégiées par la documentation écrite, car le détail de certains processus (comme la formation des systèmes économiques ou d'une culture populaire commune) n'est jamais éclairé directement par les sources médiévales, si pléthoriques soient-elles.

${ }^{36} \mathrm{Cf}$, Stéphane Boissellier, «The portuguese territory before modern-day Portugal: roots or precedents? A geo-historical reflection / Le territoire portugais avant le Portugal: des racines ou des précédents? Essai de réflexion géo-historique», Imago temporis. Medium Aevum, 3 (2009), p. 45-66 et 390-407.

${ }^{37}$ Précédé mais non pas provoqué: la concurrence entre princes ou les rapports hiérarchiques entre diocèses sont des phénomènes hautement volontaristes, avec une dimension symbolique très forte, qui n'agissent pas de la même façon que des flux matériels, un réseau routier ou un encadrement administratif. 
portugais qui concerne l'historien, si tant est que les deux soient dissociables ${ }^{38}$. Or, la compréhension de la genèse du Portugal a progressé en intégrant toujours plus d'acteurs dans la réflexion. Certes, les rois et les élites jouent un rôle capital dans la guerre, qui est le principal vecteur de constitution de l'espace portugais. Et le royaume du Portugal est, comme toute institution, une construction politique, nécessitant une concentration du pouvoir décisionnaire; de ce fait, l'historiographie a été, depuis la Monarquia lusitana, et reste encore focalisée par l'action des dirigeants suprêmes, à commencer par les monarques. Mais le royaume, s'il fournit un cadre référentiel indispensable à l'action de certaines forces (et à l'étude historique elle-même, qui ne peut pas embrasser la totalité de l'humanité...), ne résume pas et ne doit pas occulter la société «portugaise» - et l'on voit bien l'ambiguïté réductrice de ces qualificatifs nationaux ${ }^{39}$. Nous nous plaçons donc d'un point de vue en contradiction avec l'ethnogenèse proposée pour expliquer la formation des royaumes barbares du haut Moyen Âge ${ }^{40}$.

Sans méconnaître le rôle moteur des élites - qui fascinent tant les historiens actuels (notamment français) des sociétés médiévales - et particulièrement l'action de cette nouvelle noblesse féodale des infanções, dont la mise en lumière constitue un des apports capitaux de J. Mattoso, il faut prendre en compte tous les hommes ayant constitué la communauté de destins qu'est le Portugal; leur action est forcément diffuse, contradictoire, impossible à lire directement et à qualifier à travers une documentation atomisée, mais c'est à l'historien d'en restituer le sens - au lieu de se replier paresseusement sur l'explicite et l'incontestable. Dans cette perspective d'une histoire sociale embrassant «le plus grand nombre», il convient donc d'étudier cette communauté comme le fruit d'une construction collective, par la rencontre, souvent convergente mais parfois contradictoire, entre des mécanismes agissant au sein du grand

${ }^{38}$ Cette distinction constitue l'articulation principale de la mémorable somme ethnographique sur le Portugal de J. Leite de Vasconcelos.

39 Certaines actions sociales et culturelles s'arrêtent bien à des frontières (la communication dans une langue donnée, la circulation de la monnaie, l'intervention d'agents administratifs...), mais la stratification sociale, les jeux de l'échange économique, le système de valeurs morales... ne prennent pas ici des inflexions très différentes de ce que l'on observe dans le reste de la Péninsule ibérique ou même dans l'ensemble de l'Occident.

${ }^{40}$ Cette thèse de l'ethnogenèse propose que des élites germaniques très restreintes, souvent installées pacifiquement dans l'Empire, ont fini par accéder au pouvoir et ont inventé les migrations massives des peuples dont ils étaient issus, comme véritables mythes fondateurs des Etats qu'ils ont créés; on a là la combinaison d'une nouvelle critique des sources narratives, d'un refus des «grandes invasions» et d'une valorisation du rôle des élites. 
nombre ${ }^{41}$ - mécanismes pas forcément contrôlés, qui sont souvent des «effets de système» - et des décisions prises par les élites gouvernantes.

On admet généralement que les moyens coercitifs et persuasifs, dans les sociétés pré-industrielles, limitent l'emprise directe des dirigeants sur les populations, et que, en-dehors du projet totalitaire de l'Eglise grégorienne, les responsables politiques médiévaux ne se soucient pas d'influencer certains domaines de la vie de leurs sujets (l'investissement économique, le progrès technique, la démographie, le recensement...). Certes, le conformisme (à un système de valeurs dicté d'en haut) - et peut-être même le fatalisme - des sujets conduit le plus souvent ces derniers à valider, sinon à appliquer, les choix politiques, lesquels apparaissent donc le plus souvent en harmonie avec la société et peuvent passer de ce fait pour des moteurs de ladite société. Mais il suffit d'une inertie ou, beaucoup plus rarement, d'une opposition c'est le cas pour la destitution de Sanche II - pour révéler que les masses de simples roturiers ont leur vie propre et leurs propres moteurs, qui s'imposent largement aux décideurs.

Cette participation des roturiers à la construction d'une communauté avec ou, plus probablement, sans conscience identitaire globale - est liée à de multiples facteurs. Dans cette optique, le débat sur le degré de féodalisation de la société portugaise doit plutôt être déplacé sur le degré d'introversion des communautés locales d'habitants, qu'elles soient municipales ou sous seigneurie féodale; certes, l'intermédiaire que constituent les élites dominant ces communautés contribue à mettre celles-ci en réseau, mais c'est la circulation des hommes et des biens (permise par la plasticité sociale, l'exogamie, la division du travail...) au cœur même de ces masses qui assure l'intégration dans des ensembles supra-locaux.

Dans le même sens d'une histoire la plus collective possible, j'insisterai sur une dernière voie, celle des phénomènes économiques. On sait que l'historiographie française, surtout médiéviste (J. Gautier-Dalché) et moderniste (P. Vilar), a joué un rôle moteur dans le développement de l'approche économique des sociétés ibériques médiévales; mais cette histoire, trop inféodée aux modèles des économistes théoriciens, a abouti à certaines impasses méthodologiques (obsession de la quantification) et conceptuelles (utilisation de catégories impropres aux modes de production pré-industriels). Heureusement, un nouveau

${ }^{41}$ Une simple histoire démographique (telle que Teresa Ferreira Rodrigues (coord.), História da população portuguesa. Das longas permanências à conquista da modernidade, Porto, Afrontamento, 2008) peut déjà fournir une des bases nécessaires à cette réflexion. 
courant d'histoire économique, intégrant des approches plus sociologiques et anthropologiques (Karl Polanyi, Aleksander Tchayanov), peut proposer de nouvelles conceptions; je m'en fais ici, modestement, le porte-parole. Un approfondissement de la réflexion pourrait venir du rôle organisateur de l'accumulation de capital et des échanges vénaux par les agents économiques ecclésiastiques, dans le contexte de croissance des XI-XII ${ }^{e}$ siècles. Si ce système n'est pas directement à l'origine de la fondation d'un Etat, il offre un contexte favorable aux principaux acteurs politiques de la formation progressive d'une nation $^{42}$. Or, malgré le poids de l'initiative individuelle dans la colonisation agricole (notamment dans les territoires andalous), les capitaux et la capacité organisatrice nécessaires à la croissance sont, dans un contexte «grégorien», largement mobilisés par les institutions ecclésiastiques (monastères et évêchés).

Il se trouve que ce problème, s'il n'a jamais été posé en tant que tel, bénéficie, contrairement à la plupart des phénomènes macro-économiques, d'une solide base historiographique, grâce aux nombreuses et excellentes études des temporels ecclésiastiques et des institutions diocésaines ${ }^{43}$; les résultats de ces études demandent à être réinterprétés dans un schéma global.

On peut se demander notamment dans quelle mesure les richesses captées par les clercs peuvent bénéficier à l'ensemble du corps social et peuvent être mobilisées dans la formation du royaume, notamment par les premiers monarques. La fondation de puissants monastères par ces derniers et leur intervention dans les affaires de l'Eglise (à l'origine de conflits graves à partir du

${ }^{42}$ Sur les liens entre ces divers ordres de phénomènes, voir la claire réflexion de José Mattoso, «Cluny, Crúzios e cistercienses na formação de Portugal», in. José Mattoso, Portugal medieval. Novas interpretações, Lisboa, INCM, 1992 (2éd.), p. 101-121.

${ }^{43}$ Voir les travaux, classiques ou plus récents, Le cartulaire Baio-Ferrado du monastère de Grijó (XIe-XIIIe siècles). Introduction et notes de Robert Durand, Paris, Fundação Calouste Gulbenkian / Centro cultural portugués, 1971; Maria Helena Coelho, O mosteiro de Arouca do século X ao século XIII, Arouca, Câmara municipal, 1988 (2éd.); Avelino de J. da Costa, O bispo D. Pedro e a organização da diocese de Braga. 2a edição refundida e ampliada, Braga, Ed. Irmandade de S. Bento da Porta Aberta, 1997; Iria Gonçalves, O património do mosteiro de Alcobaça nos séculos XIV e XV, Lisboa, Universidade Nova de Lisboa, 1989; Armando Alberto Martins, O mosteiro de Santa Cruz de Coimbra na Idade Média, Lisboa, Centro de história da Univ. de Lisboa, 2003; José Mattoso, "L'abbaye de Pendorada des origines à 1160", Revista portuguesa de história, VII, 1957, n. 2, p. 1-192; Idem, Le monachisme ibérique et Cluny. Les monastères du diocèse de Porto de l'an mille à 1200, Louvain, Publications universitaires de Louvain, 1968; José Augusto de Sotto Mayor Pizarro, Os patronos do mosteiro de Grijó (Evolução e estrutura da família nobre séculos XI a XIV), Ponte de Lima, Ed. Carvalhos de Basto, 1995; Carlos Guardado da Silva, O mosteiro de S. Vicente de Fora. A comunidade regrante e o património rural (séculos XII-XIII), Lisboa, Edições Colibri, 2002; Hermínia Vasconcelos Vilar, As dimensões de um poder. A diocese de Évora na Iade Média, Lisboa, Editorial Estampa, 1999. 
début du XIII ${ }^{\mathrm{e}}$ siècle) peuvent être envisagés comme un élément d'organisation économique délibérée; pour le $\mathrm{XI}^{\mathrm{e}}$ siècle, l'attitude de l'aristocratie comtale au service des rois de León et surtout de la nouvelle aristocratie locale des infanções est plus difficile à interpréter dans ce sens (puisqu'il n'est pas sûr qu'il existe un «sentiment régional» chez eux), mais elle mérite elle aussi d'être au moins interrogée, dans la mesure où les intérêts économiques des laïcs utilisent très largement les établissements religieux.

Il faut postuler que l'économie ecclésiale peut structurer les activités des hommes à grande échelle et donc créer de vastes cellules territoriales. Pour la production de biens, les monastères et les églises locales du haut Moyen Âge ont une capacité organisatrice limitée à une périphérie restreinte - ce n'est qu'au XII ${ }^{\mathrm{e}}$ siècle, après l'apparition du royaume, que de grands établissements cisterciens et canoniaux auront un temporel à l'échelle régionale. Mais c'est en tant qu'acteurs des échanges et de la monétarisation et en tant que centres de consommation que les monastères et les cours épiscopales contribuent à la réticulation des foyers économiques. Bien sûr, leurs chartriers n'éclairent pas d'éventuelles relations commerciales, et c'est seulement la logique du système qui peut fonder une telle réflexion.

Cette dernière prend d'autant plus de sens si elle est corrélée avec une problématique politique: dans quelle mesure l'organisation macro-économique des hommes - en l'occurrence par l'Eglise - peut-elle concourir à leur agglutination politique ${ }^{44}$ ? A l'échelle locale, notamment dans les modèles de l'incastellamento et de l'encellulement ecclésial, on a envisagé plutôt la relation inverse: c'est la volonté politique de rassembler les hommes qui implique une nouvelle organisation économique - autrement dit le village engendre le finage. Mais le changement d'échelle inverse la problématique: à l'échelle régionale, c'est la mise en relation des lieux de production (habitats communautaires à propriété partagée, grands domaines, monastères, villes), notamment le drainage opéré par les évêques, qui, par les échanges vénaux induits, peut créer des solidarités politiques à grande échelle, si les relations entre tous les agents économiques sont suffisamment médiatisées par des intermédiaires forts comme nous pensons que l'est l'Eglise, en collusion et en confusion avec les élites laïques supérieures.

${ }^{44}$ Dans ses nombreux travaux sur la formation de l'identité portugaise, J. Mattoso n'ignore pas le rôle de l'économie (dont il fait un des piliers du royaume, avec la centralisation politique et l'identité symbolique); mais il l'envisage surtout à travers la réalisation d'un réseau urbain, et il la lie donc fortement à la volonté politique royale (ce qui en fait un agent intervenant après l'indépendance politique). 
On revient finalement à la problématique de la territorialisation en postulant que l'ensemble des institutions ecclésiastiques existant dans les marches portugaises du royaume léonais avant l'indépendance, au $\mathrm{XI}^{\mathrm{e}}$ siècle, constitue un système économique - et non pas une construction politique définissant spatialement le territoire portugais; ceci implique que la captation par l'Eglise d'une partie des profits de la croissance générale de l'époque féodale - qui est, ici, une période de conquête extérieure et de colonisation - contribue à augmenter, voire suscite, sa réticularité spatiale et fonctionnelle.

Ces directions, les médiévistes portugais les ont déjà suivies, mais ils ont abordé les phénomènes de territorialisation, d'auto-organisation sociale et de systématisation économique (notamment la «commercialisation» des grandes masses d'acteurs) comme des œuvres conscientes réalisées par les princes, donc postérieurement à la proclamation du regnum; cette focalisation de l'historiographie sur les agents détenteurs du pouvoir suprême et surtout d'une volonté claire et globale s'explique en partie par le poids du modèle de «l'histoire nationale» et par la prudence méthodologique (la répugnance à englober les grandes masses de faits ponctuels manifestés documentairement dans un système explicatif qui ne serait pas lui-même issu des sources). Evidemment, une fois que le royaume existe, il constitue le cadre référentiel majeur, et il s'introduit, chez tous les acteurs, une volonté subjective au sein de mécanismes objectifs. 